\title{
Onset of adolescent eating disorders: population based cohort study over 3 years
}

\author{
G C Patton, R Selzer, C Coffey, J B Carlin, R Wolfe
}

\begin{abstract}
Objective To study the predictors of new eating disorders in an adolescent cohort.

Design Cohort study over 3 years with six waves. Subjects Students, initially aged 14-15 years, from 44 secondary schools in the state of Victoria, Australia. Outcome measures Weight $(\mathrm{kg})$, height $(\mathrm{cm})$, dieting (adolescent dieting scale), psychiatric morbidity (revised clinical interview schedule), and eating disorder (branched eating disorders test). Eating disorder (partial syndrome) was defined when a subject met two criteria for either anorexia nervosa or bulimia nervosa according to the Diagnostic and Statistical Manual of Mental Disorders, fourth edition (DSM-IV).

Results At the start of the study, 3.3\% (29/888) of female subjects and $0.3 \%(2 / 811)$ of male subjects had partial syndromes of eating disorders. The rate of development of new eating disorder per 1000 person years of observation was 21.8 in female subjects and 6.0 in male subjects. Female subjects who dieted at a severe level were 18 times more likely to develop an eating disorder than those who did not diet, and female subjects who dieted at a moderate level were five times more likely to develop an eating disorder than those who did not diet. Psychiatric morbidity predicted the onset of eating disorder independently of dieting status so that those subjects in the highest morbidity category had an almost sevenfold increased risk of developing an eating disorder. After adjustment for earlier dieting and psychiatric morbidity, body mass index, extent of exercise, and sex were not predictive of new eating disorders.

Conclusions Dieting is the most important predictor of new eating disorders. Differences in the incidence of eating disorders between sexes were largely accounted for by the high rates of earlier dieting and psychiatric morbidity in the female subjects. In adolescents, controlling weight by exercise rather than diet restriction seems to carry less risk of development of eating disorders.
\end{abstract}

\section{Introduction}

Eating disorders in young women are common and associated with significant mortality and morbidity. ${ }^{1}$ Lifetime risks in women have been estimated at $8 \%$ for bulimic syndromes and around 3\% for anorexic syndromes. $^{34}$ The features of eating disorders most commonly emerge in mid-adolescence, before the development of full syndromes. ${ }^{5}$ Cross sectional surveys have confirmed that eating disorders are common in adolescent women; around $0.5 \%$ have anorexia nervosa, $1 \%$ have bulimia nervosa, and $3 \%$ to $5 \%$ have subclinical syndromes. ${ }^{6} 7$

Rigorous population based studies on risks for eating disorders have been few. A recent case-control study of young adults linked dieting, psychiatric morbidity, and obesity to eating disorders. ${ }^{5}$ But because these subjects were studied on average eight years after the first appearance of symptoms, it was difficult to distinguish influences on the course of the eating disorder from factors that caused it. Case-control studies are also restricted in the evaluation of factors that vary with time, for example, dieting and psychiatric morbidity, when an eating disorder can influence both the presence of a risk factor and its recall. The interpretation of available cohort studies has been marred by incompleteness of follow up, restriction to only two measurement points, and uncertain reliability in the measurement of both eating disorders and putative risk factors. ${ }^{89}$ Many of these earlier limitations have been addressed in our prospective study of eating disorders in almost 2000 Australian secondary school students.

\section{Subjects and methods}

\section{Sample population and methodology}

Between August 1992 and July 1995, we collected data on subjects in a cohort study of adolescent health in the state of Victoria, Australia. The cohort was defined using a two stage procedure. At stage 1, we randomly selected 45 schools from a stratified frame of government, catholic, and independent schools (total number 60 905). At stage 2, a single intact class from each participating school was selected at random to constitute the wave 1 sample. At the second wave of data collection 6 months later, when the wave 1 sample had moved into year 10, a second intact class from each participating school was selected at random (fig 1). One school from the initial cross sectional survey was unavailable for study leaving a total of 44 schools.

Written parental consent for participation was sought at entry of the students into the study. The subjects completed all measures at intervals of 6 months between year 9 and year 12 (six waves). Measures were administered by 28 laptop computers. ${ }^{10}$ Subjects unavailable for follow up at school were interviewed by telephone.

\section{Measures}

Eating disorders-The branched eating disorders test was used to assess criteria for eating disorders accord-

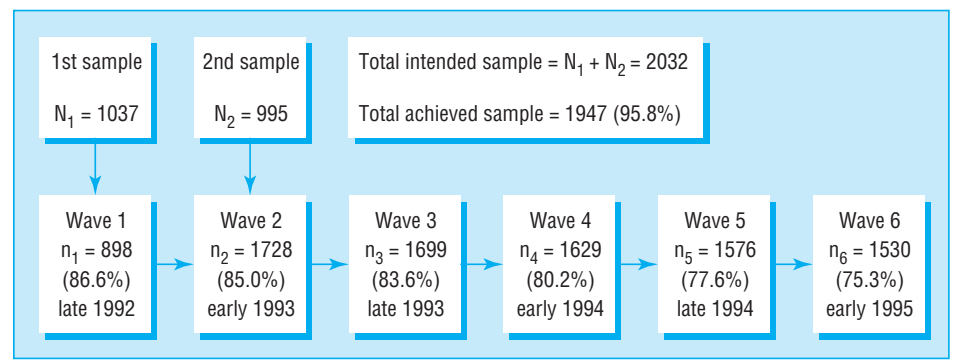

Fig 1 Participation rates of 2032 secondary school students in adolescent health cohort study in Victoria, Australia

\section{Centre for \\ Adolescent Health, \\ Department of \\ Paediatrics, \\ University of \\ Melbourne, \\ Parkville Victoria \\ 3052, Australia \\ G C Patton, \\ professor \\ R Selzer, \\ research fellow \\ Carolyn Coffey, \\ research officer \\ Clinical \\ Epidemiology and Biostatistics, Royal \\ Children's Hospital \\ Research Institute, \\ Parkville Victoria \\ 3052, Australia \\ J B Carlin, \\ associate professor \\ R Wolfe, \\ postdoctoral research \\ fellow \\ Correspondence to: Professor Patton patton@cryptic.rch. unimelb.edu.au}

BMJ 1999;318:765-8 
ing to the Diagnostic and Statistical Manual of Mental Disorders, fourth edition (DSM-IV). ${ }^{11}$ The test was designed for use in adolescent community samples and covers symptoms of eating disorders over the previous 3 months. A partial syndrome of eating disorder was defined when a subject met two DSM-IV criteria for either anorexia nervosa or bulimia nervosa. The definitions of partial syndrome using the test had high agreement with the eating disorders examination in an earlier community based validation study (sensitivity 1.0 , specificity 0.99 , and positive predictive value 0.7$).{ }^{12}$

Weight and height-Weight was measured to the nearest $0.1 \mathrm{~kg}$ with subjects in minimal school uniform, and $1 \mathrm{~kg}$ was deducted to account for clothing. Height was measured with a stadiometer to the nearest centimetre, with shoes removed. Self reported weights were used for those subjects who had left school.

Dieting and exercise-Dieting level was categorised using the adolescent dieting scale, which comprises nine items and measures three dieting strategies: calorie counting, reducing food quantities at meals, and skipping meals. ${ }^{13}$ Two types of exercise frequency were measured: daily participation in sport or a formal exercise routine. Dieting levels in schools were estimated from the rates of moderate dieting at inception of the cohort, and the schools were then divided into thirds (on the basis of dieting rates within the school) to give a measure of exposure to dieting in school peers.

Psychiatric morbidity-Mental health status was evaluated with a computerised form of the revised clinical interview schedule, which is a branched questionnaire for assessing common symptoms of psychiatric disorder in non-clinical populations. ${ }^{14}{ }^{15}$ Total scores were used to categorise subjects on four levels of psychiatric morbidity: $0-5,6-11,12-17$, and 18 or higher. A threshold of 12 or higher indicated when a doctor might be expected to have concern about a subject's mental health. ${ }^{14}$

\section{Analysis}

We analysed the data with STATA software. Estimation procedures were used with logistic regression analyses to allow for probability weighting and complex survey design. Where appropriate, confidence intervals for rates and proportions were based on methods that use Poisson or binomial distributions to allow for small numbers. We calculated incidence rates for eating disorder by dividing the numbers of incident cases by total person time at risk. Person time at risk was calculated using midpoints between relevant data waves for new cases. Multivariable analysis of prediction of a new disorder was performed with a discrete time version of the Cox proportional hazards model, which may be fitted using standard generalised linear modelling methods. ${ }^{16} \mathrm{~A}$ binary outcome was defined-that is, first onset of eating disorder-for each at risk time interval, and this was analysed using the "glm" command in STATA with the complementary log-log link function, including an offset term equal to the natural logarithm of the length of the time interval. ${ }^{17}$

\section{Results}

Overall, 1947 of 2032 students (95.8\%) completed study measures at least once during the study. Figure 1 shows the response rates of the total sample across the six waves. The sex ratio of the cohort (males $47.0 \%$ ) was similar to that in schools in Victoria at the time of sampling. ${ }^{18}$ The mean age (SD) at wave 1 was $14.5(0.5)$ years and at completion of wave 6 it was $17.4(0.4)$ years.

\section{Cross sectional profile of eating disorders}

At the completion of sampling in wave 2, 3.3\% (95\% confidence interval $2.1 \%$ to $4.5 \%$ ) of female subjects and $0.3 \%(0.1 \%$ to $1.1 \%)$ of male subjects had partial syndromes of eating disorders. Of the 30 female subjects with an eating disorder, two $(7 \%)$ had bulimia nervosa and $24(80 \%)$ had partial syndrome of bulimia nervosa giving a prevalence rate of $2.7 \%$ (1.6\% to $3.8 \%)$ at baseline in female subjects. Four of the 30 female subjects $(13 \%)$ had partial syndrome of anorexia nervosa giving a prevalence rate of $0.5 \%(0 \%$ to $1.0 \%)$ in female subjects at baseline. Three male subjects had partial syndrome of bulimia nervosa. Overall, 8\% (69/ $892)$ of females and $0.5 \%(4 / 793)$ of males dieted at a severe level, and 60\% (536) of females and 29\% (226) of males dieted at a moderate level.

We examined demographic associations with eating disorder at wave 2 using a logistic regression analysis, when the full cohort had been constituted (table 1). Eating disorders were nine times more common in the female subjects after adjustment for other covariates. Average rates of dieting in the school had a strong and dose dependent relation with eating disorders.

Subsequent to wave 2 , all new cases of eating disorder were partial syndromes of bulimia nervosa. During the study, $6.6 \%(66 / 1000)$ of female subjects and $1.2 \%$ $(11 / 947)$ of male subjects were classed as having an eating disorder on at least one occasion. Thirty three female subjects made a first transition to an eating disorder over the 2 year period from wave 2 to wave 6 , corresponding to an incidence rate of 21.8 (15.5 to $30.7)$ per 1000 person years of observation. At the immediately preceding wave, $65 \%$ of the incident cases had dieted at a moderate level and 32\% had dieted at a severe level. Eight male subjects made a first transition to an eating disorder corresponding to an incidence rate of 6.0 (3.0 to 12.0) per 1000 person years of observation. The crude incidence ratio for female subjects compared with male subjects was 3.7 (1.7 to 8.1).

Female subjects who dieted at a severe level were 18 times more likely to develop an eating disorder than those who did not diet, and female subjects who dieted at a moderate level were five times more likely to

Table 1 Adjusted odds ratios from logistic regression analysis examining cross sectional associations with eating disorders in 33 of 1728 students at completion of sampling (wave 2)

\begin{tabular}{lc} 
Variable & Adjusted odds ratios $\mathbf{( 9 5 \%} \mathbf{~ C l})$ \\
\hline Age & $1.0(0.46$ to 2.3$)$ \\
\hline Female & $8.8(2.6$ to 30$)$ \\
\hline Dieting level in school: & 1.0 \\
\hline Low & $6.7(1.3$ to 35$)$ \\
\hline Intermediate & $16(3.5$ to 75$)$ \\
\hline High & $1.2(0.39$ to 3.9$)$ \\
\hline Pustralian born & $1.3(0.54$ to 3.0$)$ \\
\hline Metropolitan school & $1.1(0.39$ to 3.0$)$
\end{tabular}


develop an eating disorder than those who did not diet (table 2). Female subjects exhibiting the highest levels of psychiatric morbidity were over six times more likely to develop an eating disorder. Age was negatively associated with risk. After adjustment for other variables, the difference between sexes was substantially reduced (relative risk 1.7, 0.67 to 4.4 ). It was not possible to test for effect modification because of the small number of events observed.

Figure 2 shows the incidence rates for eating disorders in female subjects classed according to dieting status and psychiatric morbidity 6 months earlier. Female subjects in the severe dieting group with high levels of psychiatric morbidity had a $28 \%$ chance $(14 \%$ to $56 \%)$ of developing an eating disorder within 12 months. In contrast, rates of eating disorder in female subjects in the non-dieting group with low psychiatric morbidity were exceedingly low.

\section{Discussion}

Our study showed that $8 \%$ of 15 year old girls dieted at a severe level and a further $60 \%$ dieted at a moderate level. Female subjects who dieted at a severe level were 18 times more likely to develop a new eating disorder within 6 months than those who did not diet, and over 12 months had an almost 1 in 5 chance of developing a new eating disorder. Female subjects who dieted at a moderate level were five times more likely to develop a new eating disorder than those who did not diet, and over 12 months had a 1 in 40 chance of developing a new eating disorder. In contrast, fewer than 1 in 500 subjects in the non-dieting group developed an eating disorder in the next year. Even ostensibly moderate dieting increased the risk for eating disorders, and around two thirds of new cases were in female subjects who had been in the moderate rather than severe dieting group. Although these findings suggest that dieting is a very important risk factor for adolescent eating disorder, it is arguable that for those who already diet severely a process leading to eating disorder has begun. ${ }^{13}$

Psychiatric morbidity carried independent risks for developing an eating disorder. After adjusting for previous dieting, those subjects with high psychiatric morbidity had a more than six times increased risk of developing a new eating disorder. In the high morbid-

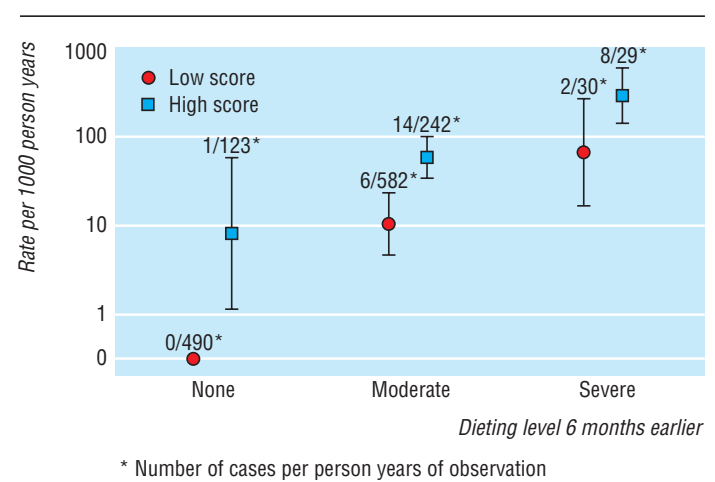

Fig 2 Incidence rates (95\% confidence intervals) for eating disorder among female secondary school students classed according to dieting level and psychiatric morbidity (low or high scores for clinical interview schedule) 6 months earlier
Table 2 Adjusted hazard ratios for discrete time survival models examining prediction of first onset of partial syndromes of eating disorder in 37 students in 2651 person years of observation*

\begin{tabular}{|c|c|c|}
\hline & $\begin{array}{l}\text { Unadjusted hazard ratios } \\
\qquad(95 \% \mathrm{CI})\end{array}$ & $\begin{array}{c}\text { Adjusted hazard ratios } \\
(95 \% \mathrm{CI})\end{array}$ \\
\hline$\overline{\text { Age }}$ & $0.38(0.25$ to 0.59$)$ & $0.48(0.28$ to 0.8$)$ \\
\hline Female & $4.5(1.9$ to 11$)$ & $1.7(0.67$ to 4.4$)$ \\
\hline \multicolumn{3}{|c|}{ Dieting status (at previous wave): } \\
\hline Minimal or non-dieting & 1.0 & 1.0 \\
\hline Intermediate dieting & 9.1 (3.1 to 26$)$ & $4.9(1.7$ to 15$)$ \\
\hline Severe dieting & 66 (20 to 211$)$ & 18 (4.9 to 67$)$ \\
\hline \multicolumn{3}{|l|}{ Psychiatric morbidity†: } \\
\hline $0-5$ & 1.0 & 1.0 \\
\hline $6-11$ & $1.2(0.33$ to 4.7$)$ & $0.86(0.23$ to 3.2$)$ \\
\hline $12-17$ & $5.4(1.9$ to 15$)$ & $3.2(1.2$ to 8.8$)$ \\
\hline $18+$ & $16(6.8$ to 36$)$ & 6.6 (2.6 to 17$)$ \\
\hline \multicolumn{3}{|c|}{ Body mass index category at previous wave: } \\
\hline$<25$ centile & 1.0 & 1.0 \\
\hline $25-75$ centile & $1.0(0.21$ to 4.4$)$ & 0.63 (0.14 to 2.8$)$ \\
\hline$>75$ centile & $2.5(0.60$ to 11$)$ & $1.1(0.26$ to 5.0$)$ \\
\hline Daily sport & $0.92(0.45$ to 1.9$)$ & 1.5 (0.69 to 3.1$)$ \\
\hline Australian born & $0.60(0.26$ to 1.4$)$ & 0.57 (0.23 to 1.4) \\
\hline Parents divorced & $1.6(0.74$ to 3.6$)$ & $1.2(0.52$ to 2.6$)$ \\
\hline \multicolumn{3}{|l|}{ School dieting level: } \\
\hline None & 1.0 & 1.0 \\
\hline Moderate & $1.6(0.68$ to 3.9$)$ & 1.2 (0.49 to 3.1$)$ \\
\hline Severe & 2.0 (0.90 to 4.7$)$ & 1.1 (0.44 to 2.8$)$ \\
\hline Metropolitan school & 1.0 (0.48 to 2.2$)$ & 0.84 (0.36 to 2.0 ) \\
\hline
\end{tabular}

${ }^{*}$ Four subjects not included as data from previous wave missing through non-participation. †According to revised clinical interview schedule.

ity group, $6 \%$ of female subjects developed an eating disorder within 12 months compared with $<1 \%$ of those in the low morbidity group. Female subjects in the severe dieting group with high levels of psychiatric morbidity had a greater than 1 in 4 chance of developing an eating disorder within 12 months.

One notable strength of our study was the multiwave design, which permitted evaluation of risk factors that vary with time (dieting, psychiatric morbidity, exercise). Other strengths derived from using a representative cohort, having high initial participation rates and low attrition rates. As school retention rates were $98 \%$ in the year of initial sampling, our cohort closely represented the broader adolescent population. ${ }^{18}$ The problem of adequately defining dieting and subclinical eating disorder was addressed through the use of standardised and reproducible measures linked to DSM-IV criteria. ${ }^{11}$ A prevalence estimate of just over $3 \%$ for partial syndromes at the start of the study is similar to reports of earlier school based samples. ${ }^{6}$ Rates of anorexia nervosa were, however, low compared with one study that used weight records in case identification but similar to those found in earlier surveys on the basis of self reported questionnaires. ${ }^{7}$ Sensitivity to questions about weight and eating may lead to non-participation in surveys by subjects with anorexia nervosa, so that despite high overall study participation, anorexic syndromes may be underrepresented. ${ }^{14}$

A sex ratio of around 4:1 for the development of eating disorder was lower than the initial prevalence ratio of around $10: 1$, raising a possibility that differences in both inception rates and early course of the eating disorder contribute to the difference in prevalence between the sexes. More importantly, the hazard ratio for sex in relation to onset of eating disor- 
- Adolescent females who diet at a severe level are 18 times more likely to develop an eating disorder than those who do not diet, and those who diet at a moderate level are five times more likely to develop an eating disorder

- High levels of psychiatric morbidity in females increase the risk of developing eating disorders by sevenfold

- Around two thirds of new cases of eating disorder arise in females who have dieted moderately

- The predominance of eating disorders in females is largely explained by the higher rates of earlier dieting and psychiatric morbidity

- Daily exercise seems to be a less risky strategy for controlling weight in adolescents

der was small after adjustment for dieting and psychiatric morbidity; both strongly associated with being a female subject. This suggests that a predominance of bulimia nervosa in female subjects arises largely from differential rates of adolescent dieting and psychiatric morbidity.

Dieting by children and adolescents, rather than other means of weight control, is likely to remain a much debated issue as obesity in young people increases. ${ }^{19}$ Previous reports have suggested that a risk factor for eating disorders is participation in sports, particularly those that require thinness, such as gymnastics. ${ }^{20}{ }^{21} \mathrm{In}$ our study, however, daily participation in sport did not raise risks above those already associated with dieting, suggesting that food restriction associated with specific sports underlayed the reported association. In adolescent weight control, promotion of exercise rather than restriction of dietary intake may prove less of a risk in the development of eating disorders.

Contributors: GCP, the principal investigator on the Victorian adolescent health cohort study, initiated the study, took part in the development and selection of study measures, and undertook data analysis and writing of the paper; he will act as guarantor for the paper. RS took part in the development, validation, and selection of study measures, and contributed to data analysis and editing of the paper. CC supervised data collection and data cleaning and analysis and contributed to both the writ- ing and editing of the paper. JBC oversaw the data analysis and contributed to the writing and editing of the paper. RW contributed to the data analysis as well as the writing and editing of the paper.

Funding: Victorian Health Promotion Foundation and the National Health and Medical Research Council. Competing interests:

1 King M. Eating disorders in general practice. $B M J$ 1987;17:249-53.

2 Harris EC, Barraclough B. Excess mortality of mental disorder. Br J Psychiatry 1998;173:11-53.

3 Kendler KS, Maclean C, Neale M, Kessler R, Heath A, Eaves L. The genetic epidemiology of bulimia nervosa. Am J Psychiatry 1991;148: 1627-37.

4 Walters EE, Kendler KS. Anorexia nervosa and anorexic-like syndromes in a population-based female twin sample. Am J Psychiatry 1995;152:6471.

5 Fairburn CG, Welch SL, Doll HA, Davies BA, O'Connor ME. Risk factors for bulimia nervosa: a community-based case control study. Arch Gen Psychiatry 1997;54:509-17.

6 Johnson-Sabine E, Wood K, Patton G, Mann A, Wakeling A. Abnormal eating attitudes in London schoolgirls-a prospective epidemiological study: factors associated with abnormal response on screening questionnaires. Psychol Med 1988;18:615-22.

7 Rastam MV, Gillberg C, Garton M. Anorexia nervosa in a Swedish urban region. Br J Psychiatry 1989;155:642-6.

8 Patton GC, Johnson-Sabine E, Wood K, Mann AH, Wakeling A. Abnormal eating attitudes in London schoolgirls-a prospective epidemiological study: outcome at twelve months. Psychol Med 1990;20: 382-94.

9 Hsu LKG. Can dieting cause an eating disorder? Psychol Med 1997; 27:509-13.

10 Paperny DM, Aono JY, Lehman RM, Hammar SL, Risser J. Computer-assisted detection and intervention in adolescent high-risk health behaviors. J Pediatr 1990;116:456-62.

11 American Psychiatric Association. Diagnostic and statistical manual of mental disorders, 4th ed. Washington, DC: American Psychiatric Association, 1994.

12 Selzer R, Hamill C, Bowes G, Patton G. The branched eating disorders test: validity in a non-clinical population. Int J Eat Disord 1996;20:57-64.

13 Patton GC, Carlin JB, Shao Q, Hibbert ME, Rosier M, Selzer R, et al. Adolescent dieting: health weight control or borderline eating disorder? $J$ Child Psychol Psychiatry 1997;38:299-306.

14 Lewis G, Pelosi AJ. The manual of CIS-R. London: Institute of Psychiatry, 1992.

15 Lewis G, Pelosi AJ, Glover E, Wilkinson G, Stansfeld SA, Williams P, et al. The development of a computerised assessment for minor psychiatric disorder. Psychol Med 1988;18:737-45.

16 Kalbfleisch JD, Prentice RL. The statistical analysis of failure time data. New York: John Wiley, 1980.

17 Carlin JB, Wolfe R, Coffey C, Patton GC. Analysis of binary outcomes in longitudinal studies using weighted estimating equations and discretetime survival methods: prevalence and incidence of smoking in an adolescent cohort. Stat Med 1999 (in press.)

18 Australian Bureau of Statistics. Australia's young people. Canberra: Australian Government Publishing Service, 1993.

19 Gidding SS, Bao W, Srinivasan SR, Berenson GS. Effects of secular trends in obesity on coronary risk factors in children: the Bogalusa health study. J Paediatr 1995;127:868-74.

20 Leon GR. Eating disorders in female athletes. Sports Med 1991;12:219-27.

21 French SA, Perry CL, Leon GR, Fulkerson MA. Food preferences, eating patterns, and physical activity among adolescents: correlates of eating disorder symptoms. J Adol Health 1993;15:286-94.

(Accepted 29 January 1999)

\section{One hundred years ago The piano as a cause of neuroses}

All-except perhaps teachers of music-will agree that at the present day the piano is too much with us. It is one of the drawbacks of an advanced civilisation, and if we are often tempted to pity those who lived in the dark ages before the dawn of the electric light and when the automobile was not, our satisfaction at the superior graciousness of our state may be tempered by the recollection that they were free from the everlasting tinkle of the piano. The maelstroms of crashing sounds which many performers think it necessary to produce as proof of their skill jar the delicate apparatus of the nervous system to a degree, that, in irritable persons, might have serious consequences if they were compelled to undergo the torture frequently. It was doubtless after an experience of the kind that Theophile Gautier defined music as the most costly of noises. Sir James Paget once took the trouble to calculate the number of muscular movements executed by a skilled player in a single performance. But who shall count the thrills, the pangs, the multitudinous shocks which a sensitive person suffers from the pyrotechnic display of a pianist who sets himself to tickle the ears of the groundlings? A lady boasted to Dr. Johnson that a piece which her daughter had just played was difficult. "Madam," replied the sage, "I wish it had been impossible." (BMJ 1899;i:988) 